\title{
Development of a liquid based deformable mirror
}

\author{
Edgar M. Vuelban, Nandini Bhattacharya, and Joseph M. Braat \\ Optics Research Group, Department of Imaging Science and Technology \\ Delft University of Technology Delft, The Netherlands
}

\begin{abstract}
Recently, a new type of liquid based deformable mirror has been proposed and demonstrated. The device consists of an array of vertically oriented open capillary channels immersed in a pool of two immiscible liquids and a freefloating reflective membrane, which serves as the reflecting surface. Liquid surface and membrane deformations are facilitated by means of electrocapillary actuation that induces upward or downward flow of liquid inside the capillary. This electrocapillary movement of liquid can be individually controlled. The advantages of this proposed device include high stroke dynamic range, low power dissipation, high number of actuators, fast response time, and reduced fabrication cost. The device is mainly suitable for dynamic wavefront correction. We present some aspects of the modeling of the device.
\end{abstract}

Keywords: deformable mirror, high order correction, electrocapillary, liquid

\section{INTRODUCTION}

A deformable mirror (DM) is an integral part of an adaptive optics (AO) imaging system. For astronomical application, the demands for a deformable mirror with high number of actuators, large stroke dynamic range, large frequency bandwidth, and cheaper fabrication cost are steadily on the rise due to the impending plans of building large telescopes that incorporate AO systems. These demands are unrealizable using conventional deformable mirrors that are based on thin glass plate or clamped membrane. Deformable mirrors that are based on liquid deformation could offer some advantages (e.g., high number of actuators, large stroke, initial flat surface, and reduced fabrication cost) compared to conventional solid mechanical DMs. The use of liquid as a deformable medium is not well explored and only a few research efforts have been done on liquid deformable mirrors.

Recently, a liquid deformable mirror (LDM), based on electrocapillary actuation of a thin layer of liquid covering a substrate, which contains an array of vertical capillaries, has been proposed and demonstrated. ${ }^{1}$ The basic idea behind the novel design is that as liquid flows inside the capillary it subsequently pushes the liquid layer that covers the substrate and in consequence a surface bump is created. Such capillary flow can be controlled by electrostatic means. Figure 1 shows the design concept of a electrostatically controlled liquid deformable mirror. The design concept was tested using a test device, shown in Figure 2.

We present here some aspects of the modeling of the proposed LDM. The main issues addressed in the modeling are that of the influence function, dynamic deformation of the floating membrane and surface wave generation and extinction. The modeling aspect of electrocapillary rise has been presented previously. ${ }^{2}$ The influence function of the DM is one of the most important parameters that determines the spatial resolution achievable for a given DM mirror. A finite element method is used to determine the shape of the floating membrane when subjected to a liquid outflow from the capillary. The calculation of surface waves and the deformation of the floating membrane are presented in the succeeding sections. The last section presents the outlook of the proposed LDM.

Send correspondence to E.M.V (e-mail: e.vuelban@tnw.tudelft.nl)

Advances in Adaptive Optics II, edited by Brent L. Ellerbroek, Domenico Bonaccini Calia, Proc. of SPIE Vol. 6272, 62722O, (2006) · 0277-786X/06/\$15 · doi: 10.1117/12.672870 


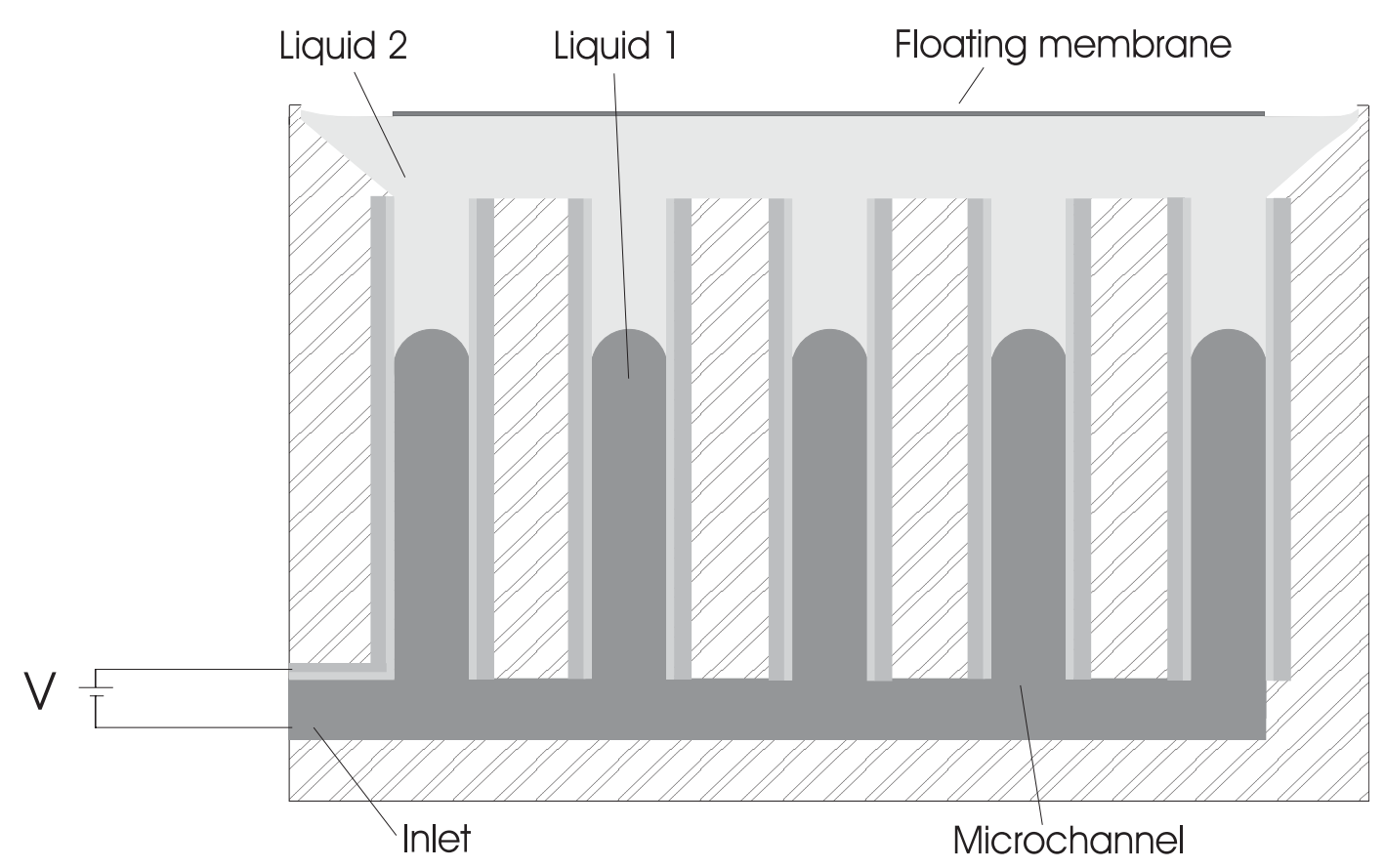

Figure 1. Device concept of a liquid deformable mirror.

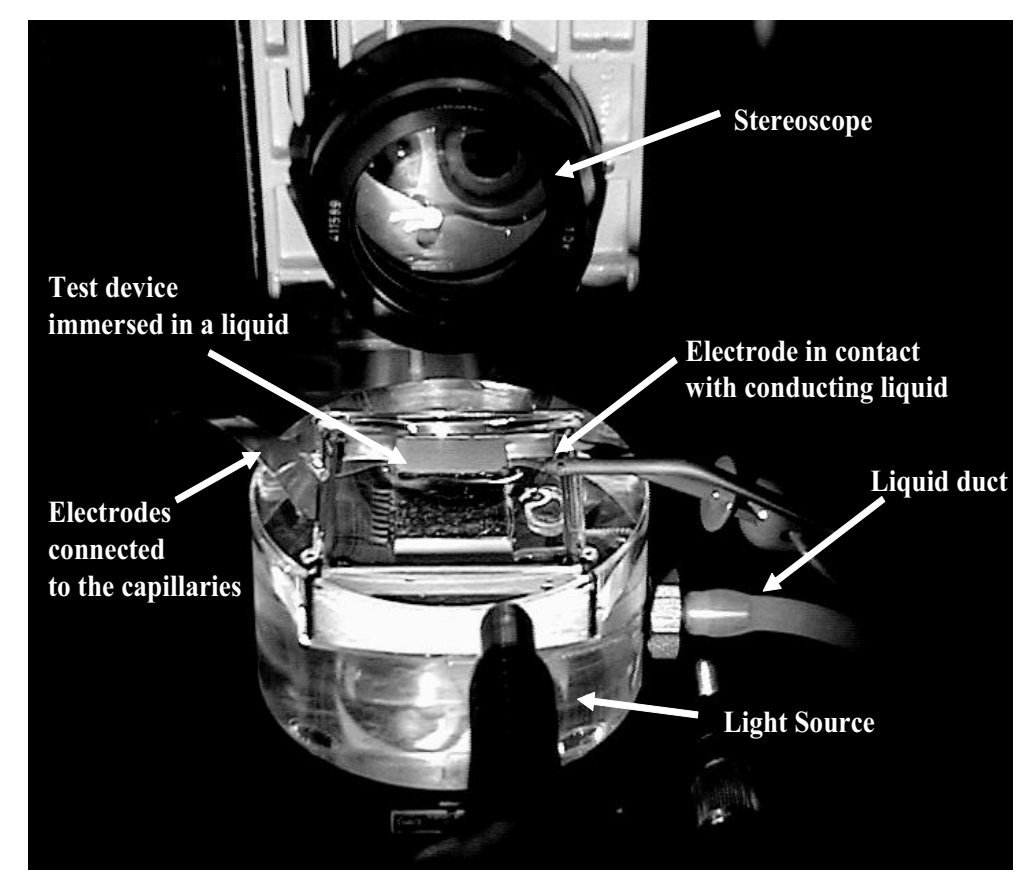

Figure 2. The experimental setup, with the test device, used to validate the design concept 


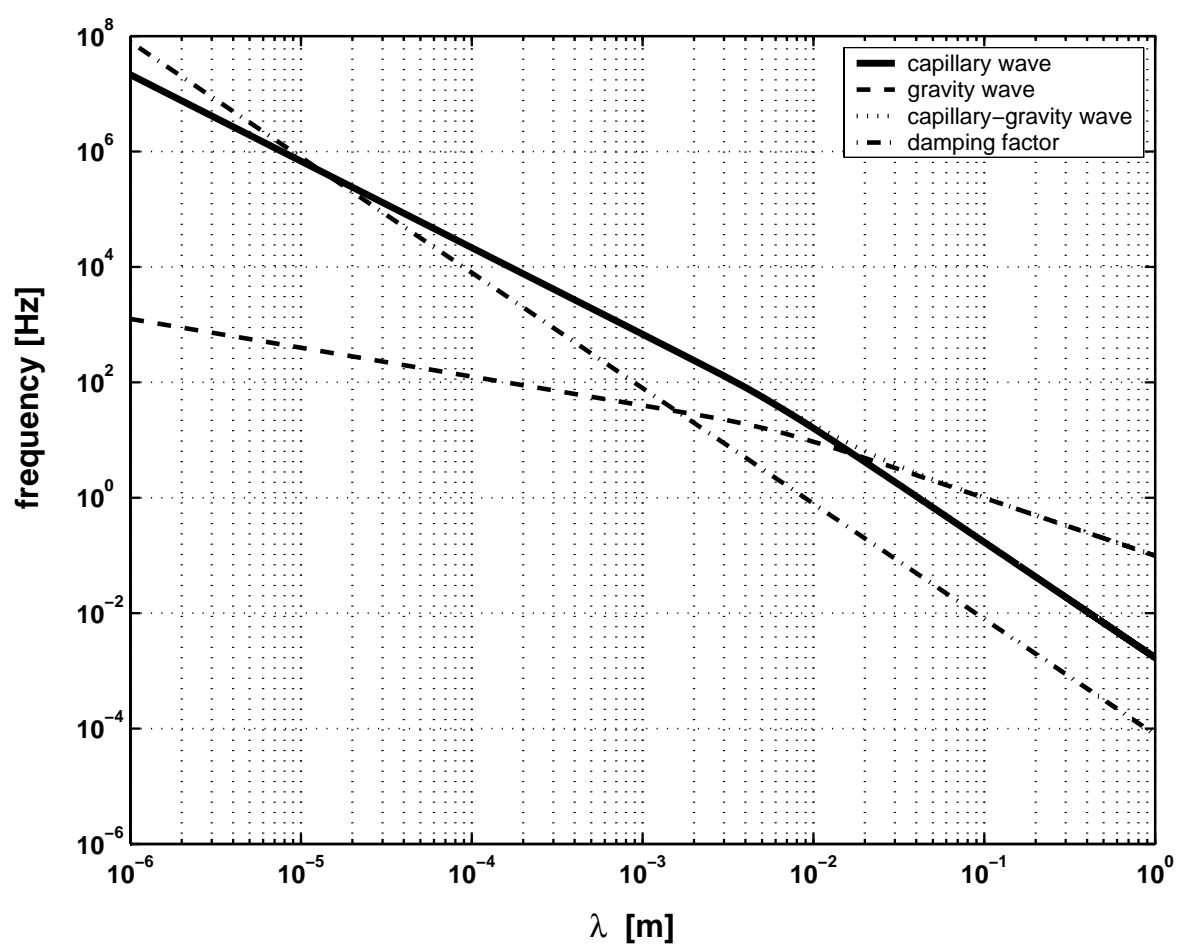

Figure 3. Surface wave dispersion relation for water.

\section{SURFACE WAVES}

One of the immediate concerns regarding the use of liquid as a deformable medium is the presence of surface waves, which can hinder the operation of the DM. The characteristics of surface waves can be studied through the well-known dispersion relation. ${ }^{3}$ Here, we consider only the case of a pure liquid. For liquids with monolayer surfactants or absorbed films, the dispersion relation is different. ${ }^{4}$ In general, for $\lambda$ (wavelength) $<<\alpha$ (capillary length), surface waves can be considered as capillary waves (characterized by a very short wavelength and high frequency) while for $\lambda>>\alpha$, gravity waves dominate. The intermediate case, where $\lambda$ is slightly less or greater than the capillary length, is called capillary-gravity wave. Surface wave propagation is accompanied by damping. The amount of damping is dependent on the kinematic viscosity of the liquid and the wavelength.

For comparison, we present the dispersion relation for water and a generic viscous liquid with varying viscosities. Both water and the generic liquid have the same thickness of $1 \mathrm{~mm}$. Figures 3 shows the dispersion curve for water waves. It can be seen from the figure that at $10 \mu \mathrm{m}<\lambda<\alpha$ (for water $\alpha=2.7 \mathrm{~mm}$ ), capillary waves dominate and its frequency is much higher than the damping factor (the kinematic viscosity of water is $1 \mathrm{cSt}$ ). At wavelengths greater than the capillary length, however, gravity waves dominate over the damping factor. Hence, waves at different wavelengths are propagated on the surface. Overdamping of waves only occurs at $\lambda<=10 \mu \mathrm{m}$. Figure 4 shows the phase velocity of water wave as a function of the wavelength. Figures 5 and 6 show the case when the kinematic viscosity of the liquid is increased by a factor of 10 and 100, respectively. The ratio between the surface tension and the density of the generic liquid is $2.5 \times 10^{-5}$. Increasing the viscosity by a factor of 10 results to the increase of the damping factor. From figure 5, it is evident that capillary waves are overdamped while gravity waves prevail at larger wavelengths. Gravity waves can be damped, until at a certain wavelength, by further increasing the viscosity of the liquid (see figure 6). 


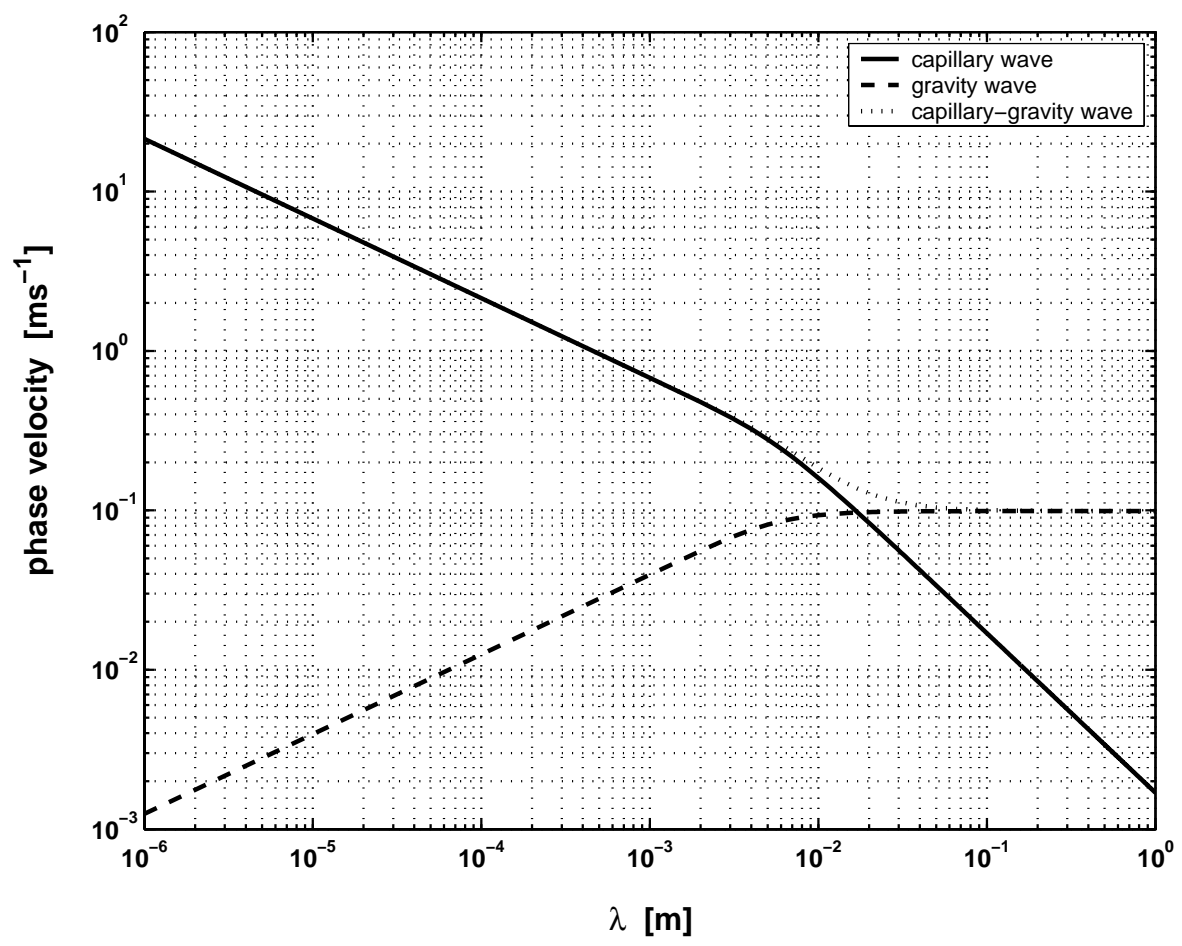

Figure 4. Phase velocity of water wave.

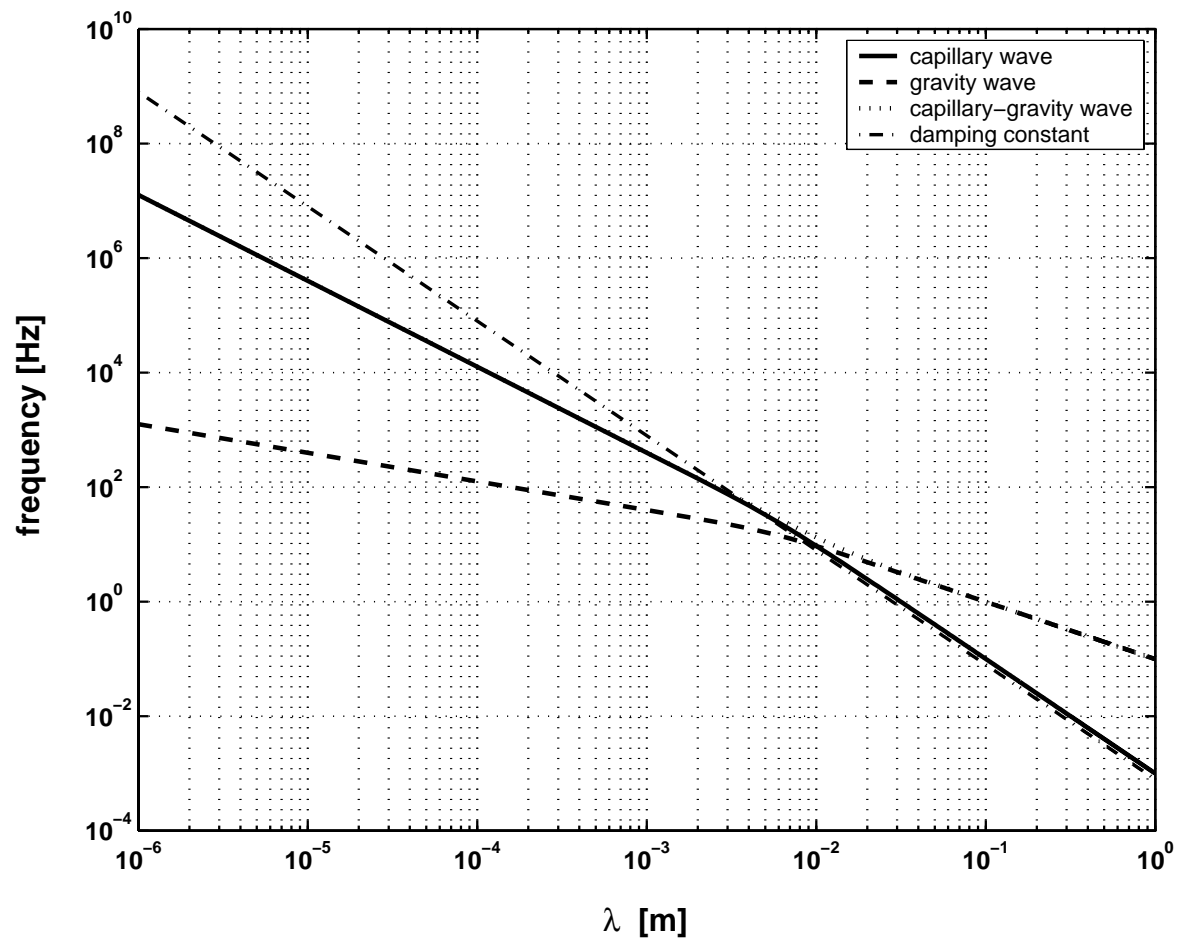

Figure 5. Surface wave dispersion relation for a generic liquid $(\nu=10 \mathrm{cSt})$. 


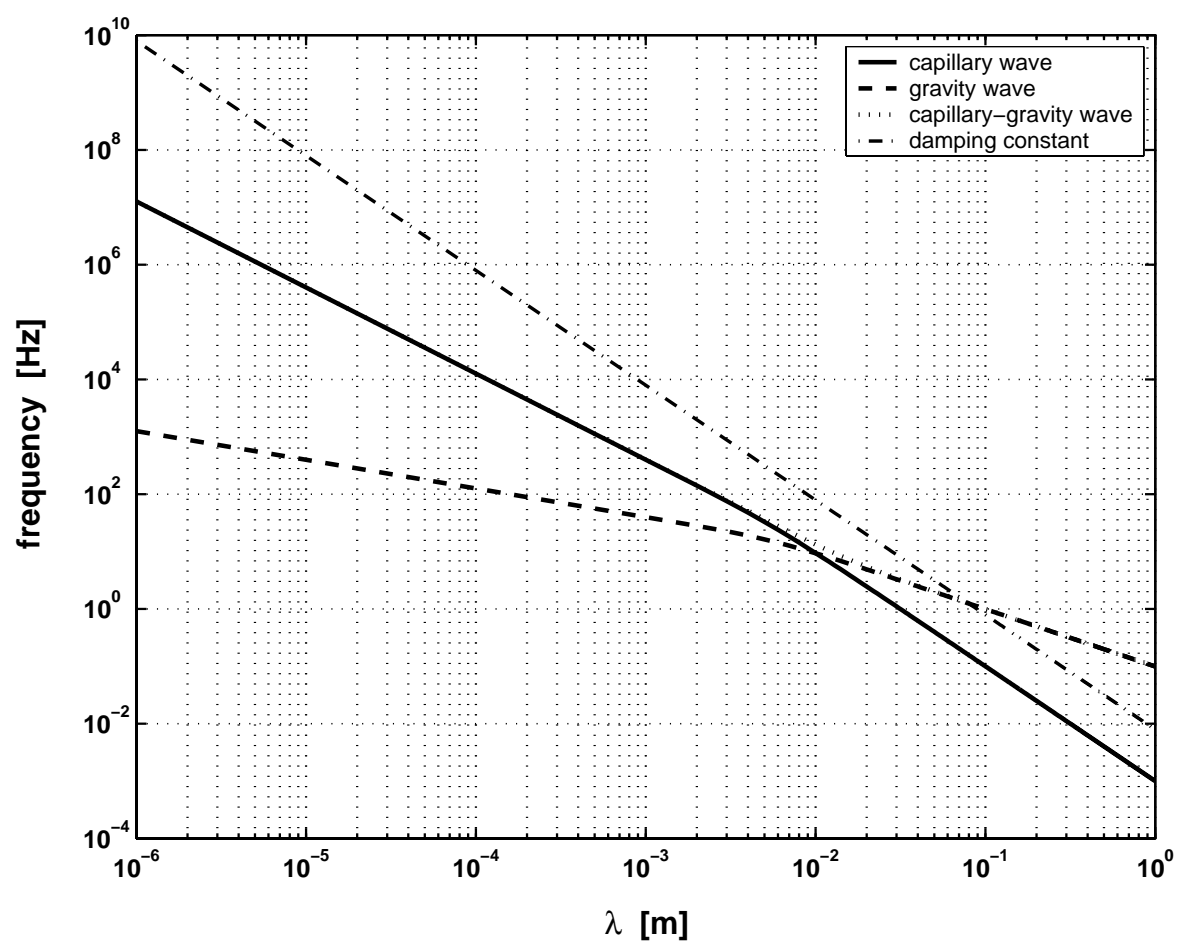

Figure 6. Surface wave dispersion relation for a generic liquid ( $\nu=100 \mathrm{cSt}$ ).

\section{FLOATING MEMBRANE DEFORMATION}

A finite element software package ${ }^{5}$ was used for the modeling of the deformation of the floating membrane and its dynamic behavior. Figure 7 shows the sub-domain definitions and the boundary conditions imposed on the model geometry. We used the ALE approach ${ }^{6}$ to determine the shape of the deformed floating membrane and its subsequent dynamic behavior. Basically, the modeling involves three distinct tasks: 1)computation of the fluid flow, 2) computation of the stresses, due to the flow, on the membrane and 3) computation of the membrane deformation and the corresponding mesh movement. For the boundary conditions on the liquid domain, we imposed a no-slip condition on the walls (boundaries 7 and 9) of the capillary as well as on boundaries 2 and 10 . At the wall of the container (boundaries 1 and 13) a slip condition is imposed. We define an inlet velocity on boundary 8 with a temporal Gaussian profile $\left(\mathrm{v}=a_{0} * \exp \left(-\left(\mathrm{t}-t_{o}\right)^{2} / \sigma\right)\right.$, with $\left.t_{o}=5 \mathrm{~ms}\right)$. The deformation profile of a $1 \mu \mathrm{m}$ thick membrane $\left(\mathrm{E}=3 \times 10^{9} \mathrm{~Pa}\right)$ at $\mathrm{t}=1 \mathrm{~ms}$ is shown in figure 8 . The profile can be well fitted by a Gaussian curve. The resulting dynamic behavior of the deformation of the floating membrane on top of water is presented in figure 9. In figure $9 \mathrm{a}$, the deformation is at $\mathrm{t}=1 \mathrm{~ms}$. The succeeding deformations (b-f) are on a $10 \mathrm{~ms}$ interval. Th liquid velocity field is also shown (light grey surface plot) in the figure. Figure 10 shows the deformation of the same membrane on top of a viscous liquid $(\nu=5 \mathrm{cSt})$ at the same time interval as in figure 9 .

\section{OUTLOOK}

The modeling results presented in this work show that surface waves would not pose any serious impediment on the operation of the device. Such waves can be substantially damped by using a liquid with the right viscosity. For the floating membrane problem, the thickness of the membrane plays a major role in terms of the widening of the profile and the subsequent dynamics of the system. Numerical simulation shows that at 1 micron thickness, the membrane with low Young's modulus follows entirely the deformation of the pure liquid. Increasing the membrane thickness results to a broader profile and thus a reduction of the DM spatial resolution. 


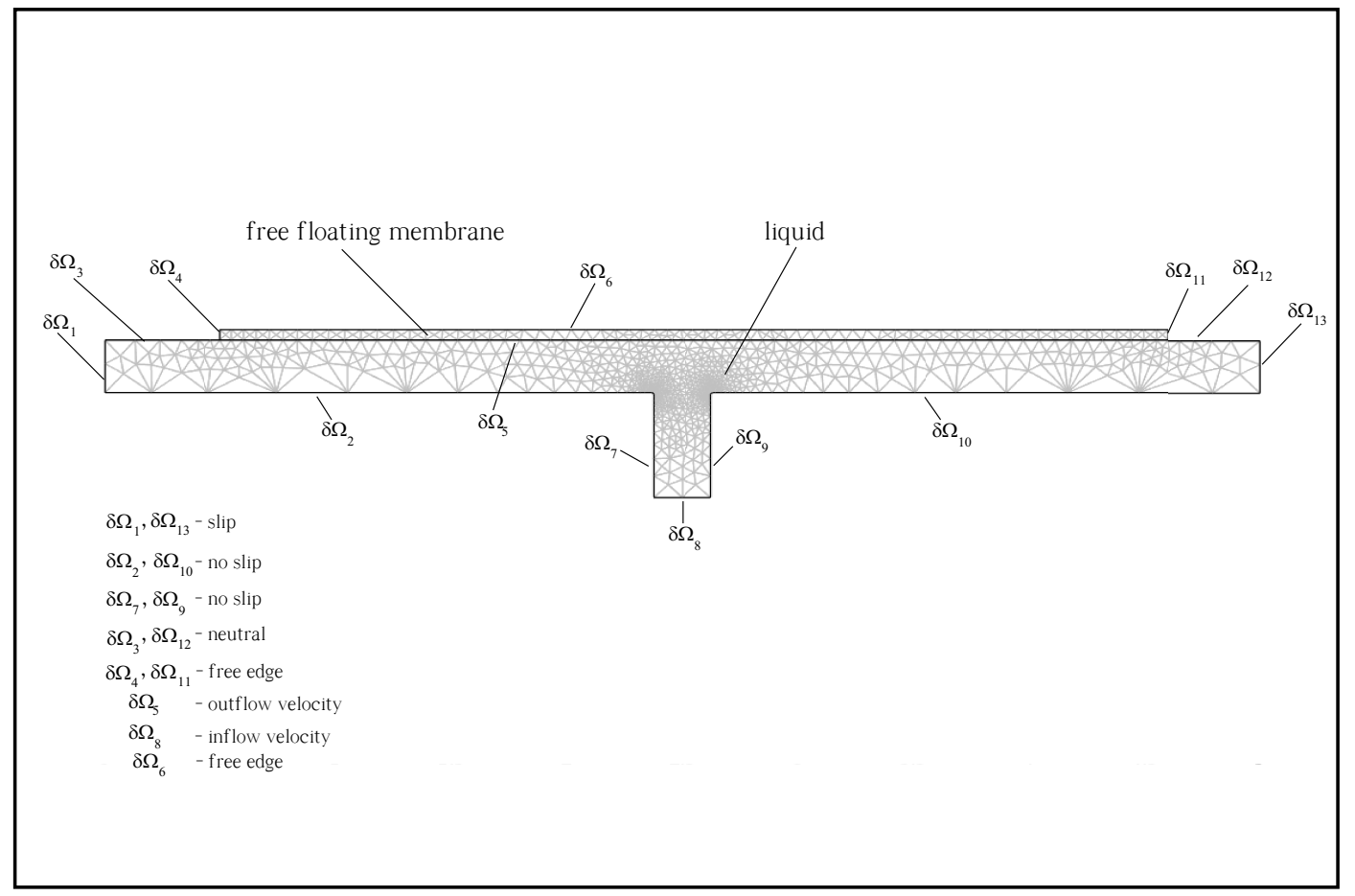

Figure 7. FEM model geometry for the floating membrane problem.

The fabrication of a prototype is still in progress. Two different platforms for fabricating the device are being implemented. The first platform is based on silicon substrate and the second one is based on printed circuit board technol0oy. These two approaches offer substantial advantages, in terms of low cost fabrication, mass production and device scaling, compared to the fabrication of conventional mechanical DMs. At the moment, there are still certain fabrication steps which are being resolved. In the very near future a demonstration of a working prototype and a comparison with the modeling results will be presented.

\section{ACKNOWLEDGMENTS}

This project is funded by the Dutch Organization for Applied Research (TNO).

\section{REFERENCES}

1. E. M. Vuelban, N. Bhattacharya, and J. J. M. Braat, Optics Letters (June 2006 issue).

2. E. M. Vuelban, N. Bhattacharya, and J. J. M. Braat, Proc. SPIE 5894, 162-169 (2005).

3. L. Landau and E. M. Lifshitz, Fluid Mechanics, Butterworth-Heinemann, Burlington, Mass., (2003).

4. V. G. Levich, Physicochemical Hydrodynamics, Prentice-Hall, New York, (1962).

5. COMSOL 3.2 User's Manual, Stockholm, 2005.

6. J. Donea, S. Giuiliani, and J. P. Halleux, Comp. Meth. Appl. Mech. Eng. 33, 689 (1982). 


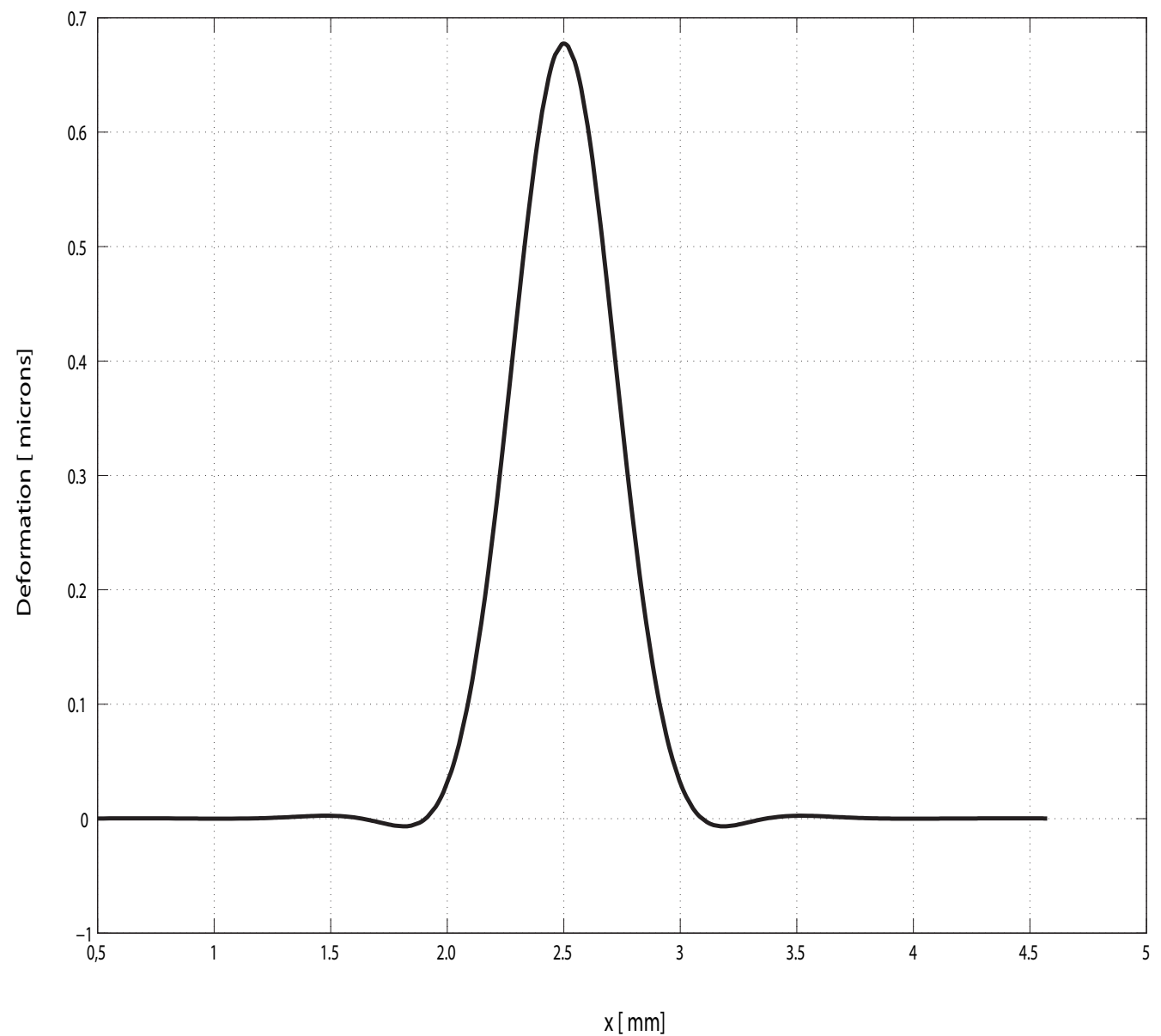

Figure 8. Deformation profile of the membrane at $\mathrm{t}=1 \mathrm{~ms}$.

Proc. of SPIE Vol. 6272 62722O-7 
a)

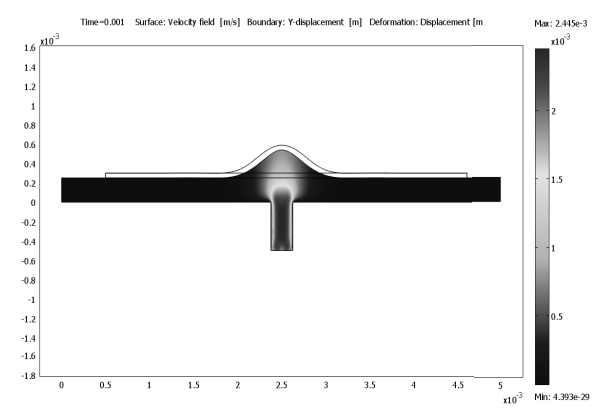

b)

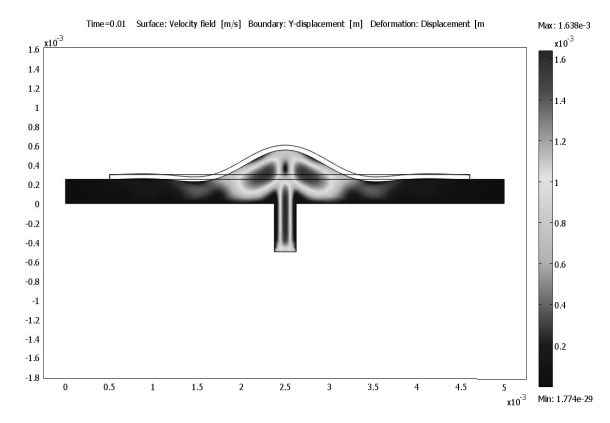

c)

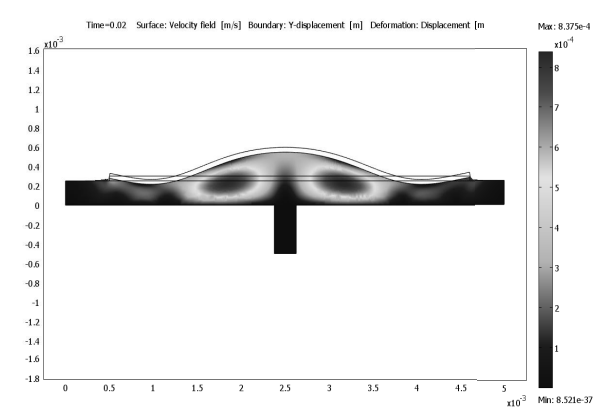

d)

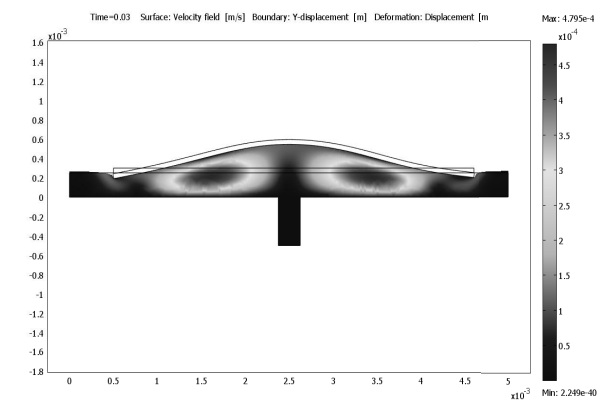

e)

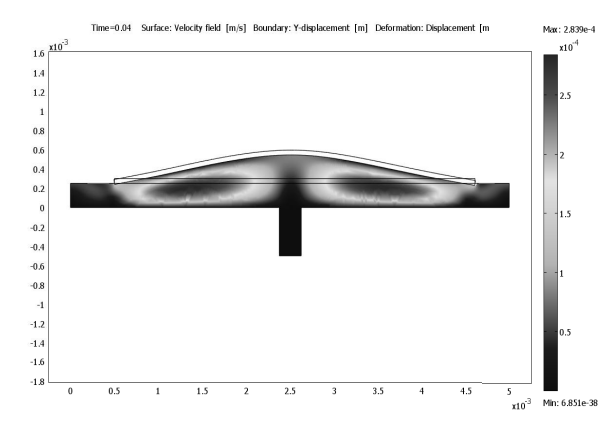

f)

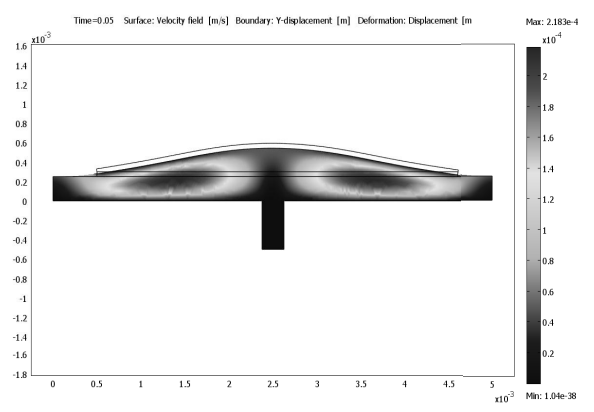

Figure 9. FEM modeling of the dynamics of the deformation of a floating membrane on water. 
a)

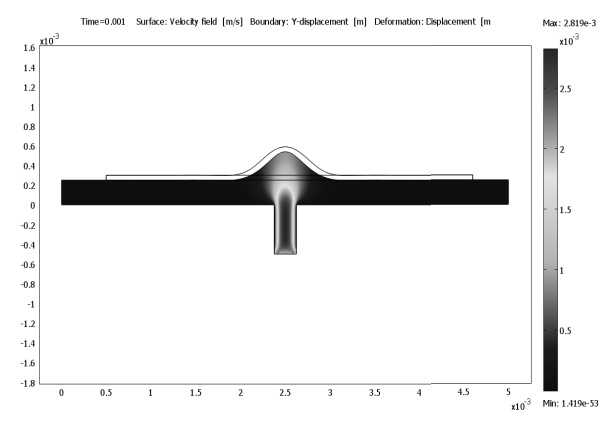

b)

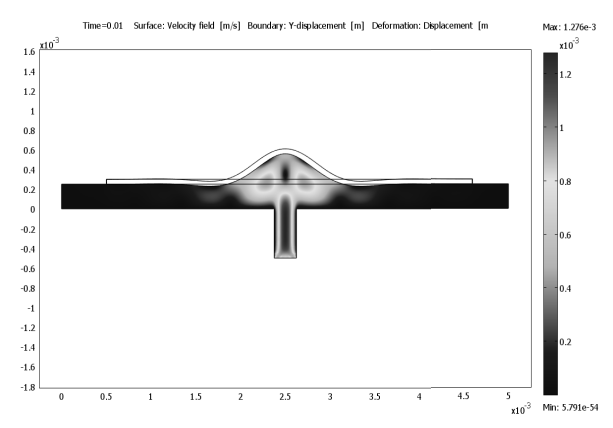

c)

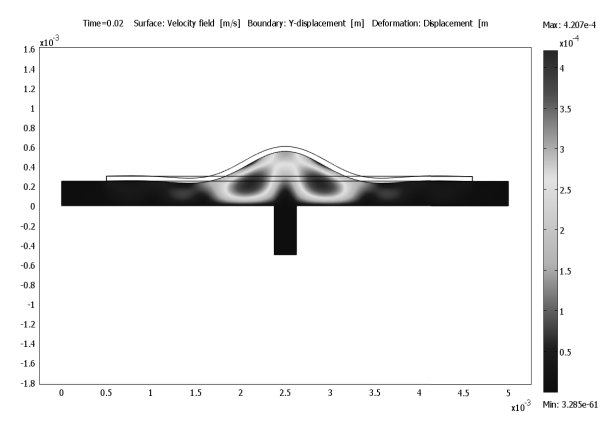

d)

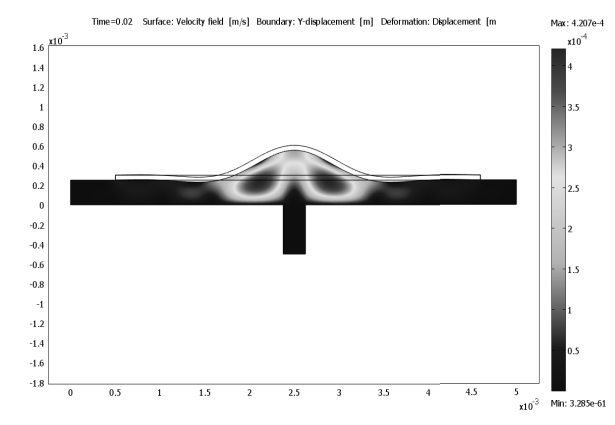

e)

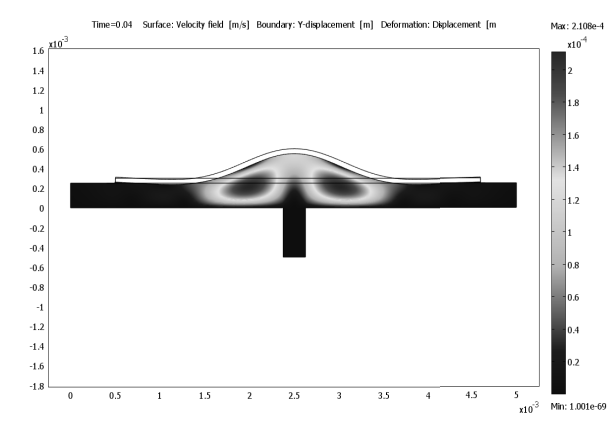

f)

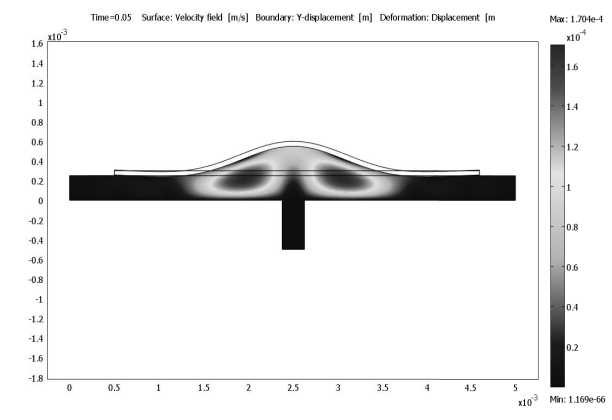

Figure 10. FEM modeling of the dynamics of the deformation of a floating membrane on a generic liquid with viscosity of 5 cSt. 